\title{
The Mentality as the Image of the Cognitive Properties of the Other
}

\author{
Klavdiya Vasilyeva \\ Peoples` Friendship University of Russia \\ Moscow, Russia
}

\author{
Osor Ochirov \\ Plekhanov Russian University of Economics \\ Moscow, Russia
}

\begin{abstract}
The article presents a probability model of gaining insight into the mentality phenomenon as a reflection of cognitive property and another mind's abilities. The authors establish a point that mentality is a result of the external evaluation since it is not self-reflected by its subject. The mentality is presented not to the subject (the media) of mentality, but to another (not-self); it is represented as a cognitive property of another as something non-identical to "not-self" insight into "self" conception (mentality media). Otherwise, namely, in the identity of "self" and "not-self" mentalities, it (the mentality of the media) is not detected. In ontological terms, "non-reflective" mentality is otherness; it remains outside of the subject. In this sense, the "nonreflective" part of the mentality is an alienated form of the neuro-mental subject organization. Substantially belonging to subject. It, nevertheless, is "alienated" from it. And in the cognitive terms, it is "assigned" by another as it is obvious to another. This reflects a sort of duality, dichotomy and the paradox of mentality.
\end{abstract}

Keywords-mentality; neuro-psychic organization; denotation; connotation; the plan of immanence; the plan of transcendence

\section{INTRODUCTION}

Scientists of today form a general opinion that mental layers are pre-reflective mechanisms, and, consequently, they cannot be principally examined in cognitive terms. In ontological terms, mentality is reduced to pre-reflective and, consequently, to unconscious. However, it is obvious to every impartial person that the necessity to structurally divide the phenomenon of mentality to its conscious and unconscious components has become a scientific reality.

\section{Mentality: The Duality OF CONSCIOUS AND UNCONSCIOUS}

\section{A. Understanding mentality from the perspective of gnosis}

Scientists associate the term of mentality with fundamental stable structures of the individual's consciousness and social communities. It's interpreted not in the narrow sense of the word, i.e. its reflective part but in the broad sense of the word - including its content and irrational structures. As is known, consciousness expresses only conscious. Whereas unconscious is cognized indirectly, though it is not self-cognizable. It is not the subject of consciousness, but it can be the object studied by consciousness. The process of explication of unconscious processes is articulated in various aspects, from the perspective of gnosis (gnosis is a Greek word that means cognition; knowledge that means an ability to cognize items through sensory perceptions) as well. Proponents of research from the perspective of gnosis denote the most crucial peculiarity of the unconscious neuro-mental activity, on the basis of which knowledge is not infrequently emerged, which is impossible when relying on rational, logical, and the verbalized experience. Therein, they see an outpacing "activity" of unconscious before conscious. The gnostic tradition admits that, if certain psychological conditions are present, the so-called "indivisible" cognition can manifest itself; it is cognition that never fails to impress us with its grandeur. Psycho-physiological research of mental processes gives scientists reason to suppose that there is a certain unified and complex system of internal relations of psyche, certain selfhood that operates and is in constant motion and change. Scientists have come to a certain point of agreement about mentality, to wit: they have recognized that there is a relative constancy of mentality elements. Its non-reflective moments is a certain general attitude or a collective pattern of thought that has a relative constancy. In general, the mental reality of man is perceived as an open informational and functional structure. In this view, mentality is regarded as the duality of conscious and unconscious and is characterized as a spontaneously active system that has a tendency not to a simple preservation of common equilibrium based upon the surrounding reality feedback principle but to the state of synergetic equilibrium. On a meta-level, mentality can be defined as follows: mentality is dynamically functional, historically and socio-culturally driven, and qualitative definiteness of the neuro-mental organization of the individual, commonness [1].

\section{B. Denotative and connotative understanding of mentality}

The modern philosophical tradition, in particular, uses a categorical and notional terminological range of conceptual frameworks which are set in the mode of "understanding explanation". First of all, this tradition stipulates that it is necessary to establish a certain object field of the object of 
cognition (signified; denotation). This establishment is possible provided that multi-factorial meanings (significant; connotation) are simultaneously included and used. Following this research path, we believe it is possible to understand the meaning, or rather a certain meaning of the signified (in this case, it is mentality). It is necessary to clarify the following: the denotation is an objective meaning. The connotation is the sense and it characterizes the denotation. We will adhere to the categorical and notional use which is mainly adopted in logical semantics. We are inclined to favor this approach because of the subject of our research, i.e. mentality and the variety of its use in different formal expressions: a notion, a word, a term which explain a wide range of scientific, philosophical and common life problems of man. The variety is so profound that sometimes we can accede that that there is no real basis even for any external order of their use.

Modern bibliography of the problem of mentality teems with research attempts to define mentality without any necessity to establish its ontological status, i.e. without specifying a specific denotation. Herein is the peculiarity and specificity of the mental reality as the subject and object of research. For instance, in "connotative semiology" of L. Hjelmslev and R. Barthes connotation is a special indirect modus of meaning, a level of a "secondary signified" (comeaning) which is built over the direct denotative meaning of a word. Subsidiary senses are formed as a result of various factors: thinking stereotypes of the scientist himself/herself, genre conventions, etc. Therefore, it is important to highlight that the meaning as the resultant of research inquiry is a relation between the signified and the significant (i.e. between the denotation and the connotation). [2]. In this view, we can probably refer to the denotative and connotative understanding of the phenomenon of mentality. As is known, connotations are constant companions of human rational or circum-rational. As a rule, they are nowhere near the denotation (signified).

\section{MENTALITY's PlAN OF CONTENT AND FORMS OF ITS MANIFESTATION.}

\section{A. Subject and object relation}

The plan of content can be manifested indirectly through the deflection of consciousness with another consciousness, inter-subjectively; through dialogues of a great many of consciousnesses through the objectification of mental phenomena in cultural forms, in social and cultural codes, etc. Structural components of the mentality's plan of content can be classified by the degree of their manifestation and expression from the following perspectives:

- Subject and object relation;

- Inter-subjective relation;

- self-consciousness, self-expression

The subject and object relation is mainly characterized by expansion in the plan of expression, meaning the demonstration of mentality to another. It can record explicit forms of expression (self-fulfillment) of mentality - verbal, muscular and motive, emotional, behavioral and other. The inter-subjective relation can reveal many details hidden from the scientist in other cognitive structures. From the perspective of the subjects' sense of self, we identify manifestations of mentality which are not obvious to the external observer. From the perspective of selfconsciousness and self-expression, it is possible at the level of the sense of self to identify implicit manifestations of mentality, which are not obvious to another (the external observer) but are represented in the form of value systems, directed intention to something, etc.

In ontological terms, the mentality's plan of content is substantially self-fixed upon the subject as an individual medium. In this sense, mentality is impenetrable for external observation. And the subjective and objective cognitive structure is of little use here. From the perspective of any cognitive structure, the mentality's plan of expression is transcended in explicit forms, for instance, verbally. There are numerous means of indirect expression of the nonreflective part of mentality, for instance, artwork, emotions, dreams, etc. Therefore, from the perspective of the subject and object relation, explicit manifestations of mentality are the result of a mechanism of transformation of potential into actual of content into expression. However, content will, to some extent, remain terra incognita both for external observation - the cognizing subject, and for the subject who is the mentality medium since the subject itself, does not often reflect this mentality.

\section{B. Inter-subjective relation}

Mentality is also manifested at the level of an intersubjective relation. Mentality is observed only from the outside, by another man, another "self" which classifies "notself" as something different from "self" as something another. Mentality is observed in the context of an opposition relation "self" - "not-self". In such a case, "not-self", being different from "self", is perceived by the latter as something present in consciousness but not outside. Therefore, "self" assumes "not-self". "Not-self" being disarticulated "self" is, first of all, a part of the subjective reality of "self". The possibility of cognitive approximation of the latter to the essence of "notself" is rather low. "Not-self" is what left beyond the selfidentification of "self". However, the classification and disarticulation process of "not-self" is performed through the prism of the experience of "self" through a mental set that belongs to "self". And then the question arises: How identical is "self" (conscious) to objective "self" (unconscious)? If they are not identical, "not-self", as it is defined, loses its meaning and sense. Therefore, as a rule, mentality at the inter-subjective level cannot be substantially defined to the full extent.

\section{Self-consciousness, self-expression of mentality}

Let us consider the mentality's plan of content of the subject from the perspective of its self-consciousness and self-expression. It is widely accepted that "mentality is not explicitly formulated and not quite realized intellectual attitudes, habits of consciousness, which do not signify the 
plan of expression, but the plan of content of human intentions, ideas and representations" [3].

The process of self-consciousness is "consciousness for self" which is immanently manifested in relation to its own sources [4]. However, the world of ideas in pure form of its alienated, transcendent image that's represented rather multiplies - as law, morale, philosophy, etc. The objective reality, i.e. the objectified reality, is a condition of the initial phase of the thinking process. In an ideal scenario, an abstractive something from the actual given serves as the reference point for the subsequent preservation process of the reality image and simultaneously for the elimination of extrinsicality and identification of several grounds of the inward nature of the transcended. From the point of view of the process, mentality's self-fulfillment (meaning the establishment) and self-expression (transcendence) of the grounds of its own nature are performed in unity of interiorization and exteriorization. However, as a rule, interiorized external objective actions and forms of their exteriorization do not match. The similarity of their nature is possible only in an ideal scenario. During the thinking process, "self" forms a subjective relation to the subject which is natural to the sense bearing structures of self being. By separating the essences from the given, "self" objectifies the former. Therefore, a self-consciousness disarticulation attitude in pure form through the consciousness itself is lost in the vicious cycle, i.e. the plan of transcendence is not equal to the plan of immanence. In other words, mentality cannot explicitly manifest its own content. Only the implicit and internally immanent carries on preserving its self identity.

\section{UNCONSCIOUSNESS OF MENTALITY IS COGNITIVELY APPROPRIATED BY ANOTHER}

On condition that the non-reflectivity of mentality is acknowledged, mentality is a product of external assessment since at the level of commonplaceness and mundanity. It is neither reflected nor displayed by its subject. If we assume that there is a certain neuro-mental organization attributed to man as a becoming homogeneous given endowed with a continuum property, then the identification of mentality as a socio-historical and socio-cultural phenomenon begins with inter-subjective relations of a certain multitude of communities. The first attribute which breaks the homogeneous continuum will be a qualitative difference, the otherness of the objective community from the subjective one. The identification of the otherness, difference and distinction assumes that there is a quality "something" ("notself") through which this otherness, difference from "self", is identified. Therefore, from the point of view of the process, the word "mentality" does not substantiate what it names, but it is used as a quality characteristic of another subject (object).

Mentality is not reflected but formulated by its mentality medium. In this sense, it is something that manifests itself to another (other). Mentality is not presented to the subject (medium) of mentality, but to another ("not-self"), is presented as a cognition property of another as something not identical to the beliefs of "not-self" about "self" (mentality medium). Otherwise, i.e. if the mentalities of "self" and "not-self" are identical; he/she (mentality medium) is not identified. But he/she is identified for a very trivial reason - the otherness, distinctiveness of the mentality of "not-self" from the mentality of "self". In ontological terms, non-reflective mentality is the otherness; it cognitively exists beyond the subject. In this sense, the non-reflective part of mentality is an alienated form of the neuro-mental organization of the individual. Substantially (in ontological terms) belonging to the subject, it is still alienated from it. In cognitive terms, it is appropriated by another. A certain duality, dichotomy and paradoxicality of mentality are manifested in this.

Furthermore, mentality is not identical to the thoughts the subject (mentality medium) expresses about himself/herself. Mentality does not "give itself away" even to its own subject, or mentality is something which is mainly non-reflective.

Not in every instance the subject understands the true reason for his/her own intention, attitude to some action or behavior. The reason is usually obvious to the external man another. And the forms of explicit expression of own content are usually reflected. So, working only with the cognizable part of mentality, we are hopelessly far away from the truth and from the knowledge of the nature of mentality. In addition, we will deal with something else, not with mentality, since mentality includes both conscious and unconscious. Therefore, the understanding of the content of mentality which aims at individual completeness is mainly possible through the study of implicit and indirect forms of expressions which are unconscious to a full degree.

\section{Post-Modernism AND COGNITIVE FUtURITY OF MENTALITY}

Achievements of sciences about the nature of mental lead to an increase of imminence of many aspects of the subject of philosophical reflection which bears witness to the spread of the opinion that the nature of metal and its phenomena cannot be comprehended [5]. The extreme example of this tendency can be found in the philosophy of post-modernism which adopted this idea from the philosophy of irrationalism. In particular, it is brilliantly manifested in the works of $\mathrm{G}$. Deleuze and F. Guattari who argued against transcendence in favor of immanence and "preferred immanence and space over transcendence and time". In each case, when immanence is interpreted as immanent to "something", there is a shift of the plan and the concept, so the concept becomes a transcendent universal, and the plan becomes an attribute within the concept [6]. From the perspective of modernism, we can speak about the plan of imminence only when imminence is imminent only to itself. "The plan of imminence is something that should be conceivable and cannot be conceivable. Apparently, this is the very inconceivable thing in a thought [7]". We find the last remark of the post-modernists to be worthy of note. And we decided to rephrase it in terms of our discourse as follows: the plan of imminence is an indefinable definiendum, thereby relating mentality to the plan of immanence as the signified. Therefore, as experimental data, which substantiates the ontological status of mentality as the given, grows, so does the cognitive perplexity of scientists since 
"no philosophical stroke of insight can provide a univocal image of humanity". It would be better to say that as the comprehensive is being transcendently mastered, there emerges a multiplicity of sources of humanity" [8]. Whereas, the philosophical interpretation of naturalistic results is manifested in the growth of the imminence of mental.

\section{CONCLUSION}

The present reader may have some connotations as to the historically known dichotomy of "reason and faith". As well as the acknowledgement of an indisputable truth - an increase of the share of unconscious in the structure of mentality of the individual or community leads to irrational behavior and actions and, consequently, the probability that men can mutually understand each other is diminished. As is known, consciousness expresses only conscious. However unconscious is cognized indirectly, though it is not selfcognizable. It is not the subject of consciousness, but it can be the object studied by consciousness.

\section{REFERENCE}

[1] Vasilyeva K. K: "Mentality: is - onto-etnological dimension (for example, the Buryat ethnos)", Moscow, Russian World, 2002

[2] Ilya Ilyin: "Postmodernism. Glossary", Moscow, Intrada, 2001

[3] Gurevich AY: "Categories of medieval culture", Moscow, 1984

[4] Belous VG: "Russian mentality // Problems of Philosophy", 1996, number 5

[5] "The philosophy is self-culture" (interview with V.A.Lektorskim) // Vestnik MGU. Filosofiya Series, 2002-number 5

[6] Delez J., F. Guattari : "What is Philosophy?" SPb, Aleteyya, 1998

[7] Ibid.

[8] Jaspers K.: "Obschaya psychopathology”, Moscow, Practice, 1997 